\title{
A Very Late Presentation of a Right Coronary Artery Occlusion After Transcatheter Aortic Valve Replacement
}

\author{
Roberto Ramirez ${ }^{\mathrm{a}, \mathrm{c}}$, Oxana Ovakimyan ${ }^{\mathrm{b}}$, Glenmore Lasamª, \\ Kristen Lafferty ${ }^{a}$
}

\begin{abstract}
Involvement of the coronary ostia is a rare complication after transcatheter aortic valve replacement (TAVR). This complication has been more commonly described immediately after valve deployment. This is the first reported case of delayed coronary obstruction caused by TAVR 6 months after the procedure.
\end{abstract}

Keywords: Coronary obstruction; Transcatheter aortic valve replacement; Right coronary artery

\section{Introduction}

Aortic stenosis is the most common native valve disease affecting up to $5 \%$ of the elderly population, and many of these patients are considered high risk for open heart surgery. Transcatheter aortic valve replacement (TAVR) has emerged as an alternative to surgical aortic valve replacement (SAVR) for patients with severe symptomatic aortic stenosis [1]. Despite increasing experience, TAVR is still associated with unexpected complications such as paravalvular aortic regurgitation, conduction disturbances, and ostial coronary occlusion. Coronary ostia occlusion is very rare; however, in cases where this occurs, the left main coronary is more commonly affected and this complication is generally reported immediately after valve implantation [2].

\section{Case Report}

A 78-year-old man presented with acute onset of severe retrosternal chest pain associated with shortness of breath. He had a history of non-obstructive coronary artery disease and severe

\footnotetext{
Manuscript submitted May 29, 2017, accepted May 31, 2017

${ }^{a}$ Overlook Medical Center, Summit, NJ, USA

${ }^{b}$ Morristown Medical Center, Morristown, NJ, USA

${ }^{\mathrm{c} C o r r e s p o n d i n g ~ A u t h o r: ~ R o b e r t o ~ H i r a m ~ R a m i r e z, ~ O v e r l o o k ~ M e d i c a l ~ C e n t e r, ~}$ Summit, NJ, USA. Email: hiram_ramirez@hotmail.com
}

doi: https://doi.org/10.14740/cr559w aortic stenosis (aortic valve area $=0.9 \mathrm{~cm}^{2}$, peak velocity $=4.2$ $\mathrm{m} / \mathrm{s}$, mean gradient $=38 \mathrm{~mm} \mathrm{Hg}$ ) status-post successful transfemoral TAVR with $25-\mathrm{mm}$ direct flow valve 6 months prior, which was complicated by heart block requiring permanent pacemaker implantation. Non-selective coronary angiography at that time showed non-obstructive coronary artery disease with TIMI flow grade 3 (Fig. 1). Before the procedure, CT angiogram noted a coronary ostia height of $11.6 \mathrm{~mm}$, a sinus of Valsalva (SOV) diameter of $28.7 \mathrm{~mm}$ and a sinotubular junction diameter of $20.3 \mathrm{~mm}$.

At the time of current presentation, on arrival to the emergency department, the patient was found to have a blood pressure of $80 / 50 \mathrm{~mm} \mathrm{Hg}$; the remainder of his vital signs and physical examination findings were within normal limits. Electrocardiogram revealed sequential atrioventricular-paced rhythm with $7 \mathrm{~mm}$ ST elevations in inferolateral leads (Fig. 2). The patient was taken for emergent cardiac catheterization and prior to the procedure he had cardiac arrest due to ventricular tachycardia. Cardiopulmonary resuscitation was initiated and direct-current cardioversion restored normal rhythm. Coronary angiography demonstrated ostial flush occlusion of the right coronary artery (RCA) (Fig. 3). The patient's blood pressure deteriorated rapidly during the procedure and an intra-aortic balloon pump was placed. The patient had another prolonged

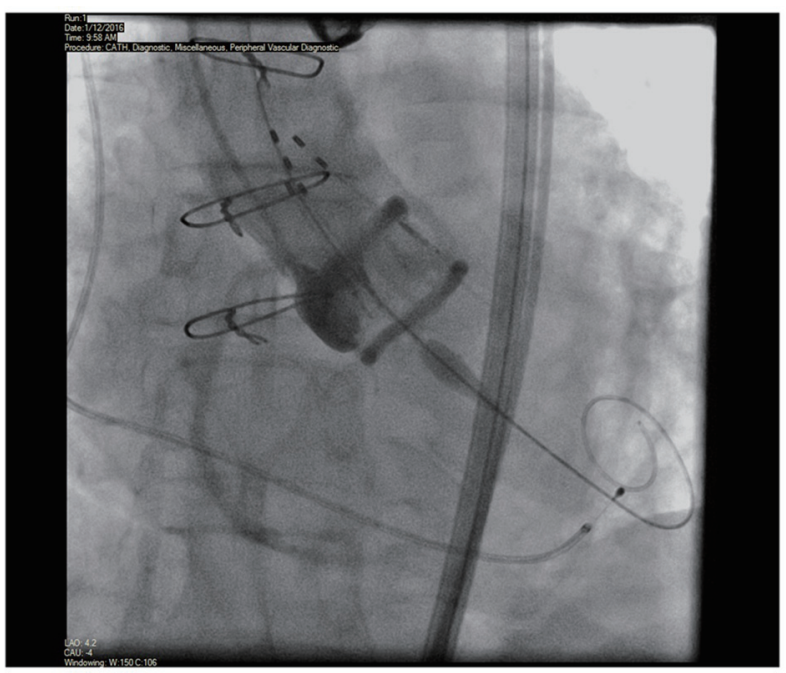

Figure 1. Non-selective coronary angiogram during TAVR showing patent right coronary artery with no significant disease. 


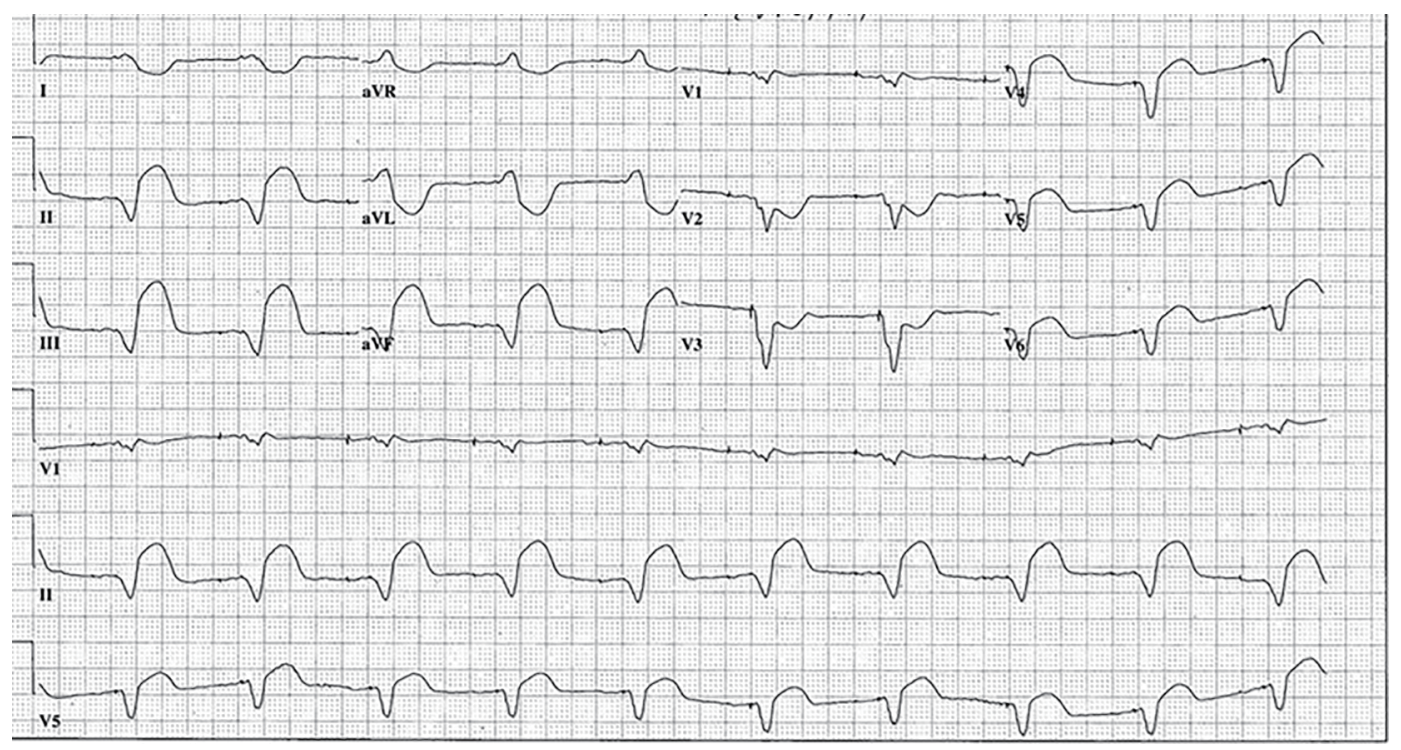

Figure 2. Electrocardiogram showing atrioventricular-paced rhythm with $7 \mathrm{~mm} \mathrm{ST} \mathrm{elevations} \mathrm{in} \mathrm{inferolateral} \mathrm{leads.}$

cardiac arrest due to ventricular fibrillation and cardiopulmonary resuscitation was resumed, unfortunately his rhythm could not be restored and the patient passed away.

\section{Discussion}

In large and medium-size case series, the prevalence of coronary artery obstruction after TAVR was $0.6-4.1 \%$ [3]. The most frequently identified obstruction is of the left main coronary artery. RCA occlusion is rare due to a higher level of RCA ostia takeoff [4]. Investigators have typically reported acute hemodynamic compromise immediately after TAVR deployment. Few cases have described migration of the aortic valve $48 \mathrm{~h}$ after implantation [2]. Only two cases reported late TAVR mi-

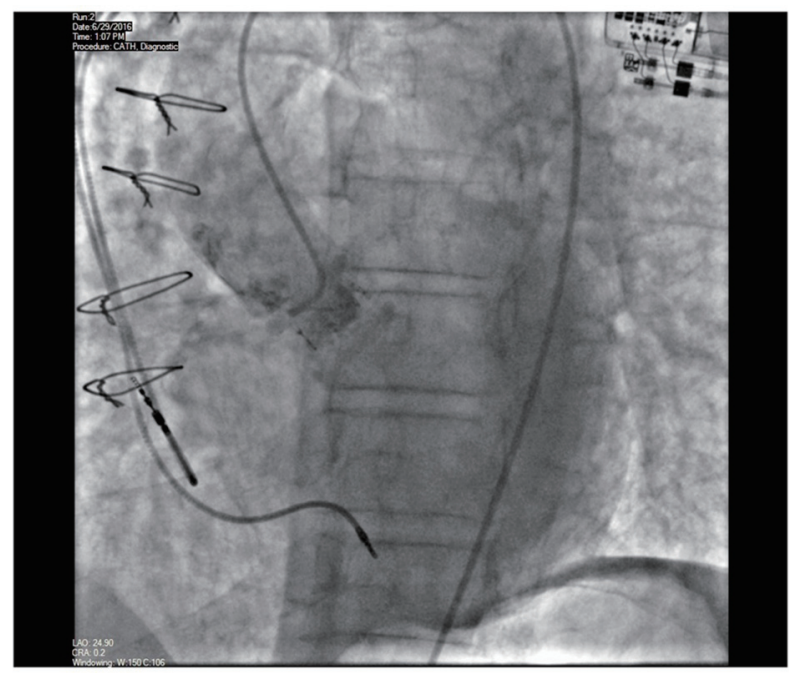

Figure 3. Non-selective coronary angiogram 6 months after TAVR showing complete occlusion of the right coronary ostia. gration up to 63 days post-procedure and in both cases the valve was displaced into the left ventricle [5, 6]. Approximately $50 \%$ of the cases presented with ST-segment changes on ECG and $25 \%$ presented with ventricular arrhythmias. The most common mechanism of coronary obstruction after valve implantation is displacement of the native calcified valve leaflet over the coronary ostium which is seen in $98 \%$ of the cases. The other possible mechanism is an impingement of the coronary ostium by the valve scaffold or leaflets [4]. Higher probabilities of occlusions occur with the balloon-expandable SAPIEN-XT valve than the Medtronic CoreValve [7], and the risk for occlusion is even higher in valve-in-valve implantation (three- to four-fold) [8]. Direct flow valve is a novel system utilizing an inflatable cuff with a conforming metal-free polymer support structure. This new valve avoids both rapid pacing of the heart during deployment, and post-dilatation after placement. In theory, this minimizes the risk of hemodynamic instability for patients allowing in situ repositioning of the valve after full deployment. This valve has not been studied as extensively as CoreValve or Sapien, therefore long-term complications are unknown.

Coronary obstruction after valve deployment is likely to be the result of anatomical factors. It is essential to have a multidetector CT angiogram in the workup of patients considered for TAVR. Proposed risk factors for coronary obstruction following TAVR include: narrow aortic root, shallow sinuses of Valsalva $<30 \mathrm{~mm}$, low position of coronary ostia $(<12 \mathrm{~mm}$ from basal leaflet insertion), pre-existing left main stem atheroma, bulky heavily calcified aortic valve cusps and larger valve sizes [9]. TAVR should probably be discouraged in patients with a coronary ostia height $<12 \mathrm{~mm}$, especially if associated with SOV diameter $<30 \mathrm{~mm}$ [4]. However, it is unproven that these factors jointly or separately lead to coronary obstruction and the cause remains unpredictable and unclear.

In our case, the patient was determined to be at high risk for coronary occlusion during TAVR. Subsequently, the direct flow valve was selected because it has the advantage of being 
repositioned or retrieved in the event of a coronary obstruction. We postulate that the direct flow valve may have migrated over time and caused a flush occlusion of the RCA ostia. Another possible explanation is that the valve partially covered the RCA ostia at the time of deployment and a thrombotic occlusion occurred at the time of the current presentation. This valve has a bulky proximal ring and a continuous covering of the frame, which may effectively obstruct the coronary ostia [10]. This is the first report of a very late occlusion of the RCA by a direct flow aortic valve 6 months after successful deployment.

Very late valve migration or thrombus formation in a coronary ostia due to a preexisting partially covered ostia is rare. However, in those patients presenting with cardiovascular compromise and a history of TAVR, these rare complications should be considered.

\section{Reference}

1. Siqueira D, Abizaid A, Arrais M, Sousa JE. Transcatheter aortic valve replacement in elderly patients. J Geriatr Cardiol. 2012;9(2):78-82.

2. Sanchez CE, Arshi A, Yakubov SJ. Managing the risk of coronary occlusion during TAVR. Effective planning for valve retrieval and coronary protection is essential. Cardiac Interventions Today. 2016;10(5):32-34.

3. Durmaz T, Ayhan H, Keles T, Aslan AN, Erdogan KE, Sari $\mathrm{C}$, Bilen E, et al. Left main coronary artery obstruction by dislodged native-valve calculus after transcatheter aortic valve replacement. Tex Heart Inst J. 2014;41(4):414-417.

4. Ribeiro HB, Webb JG, Makkar RR, Cohen MG, Kapa- dia SR, Kodali S, Tamburino C, et al. Predictive factors, management, and clinical outcomes of coronary obstruction following transcatheter aortic valve implantation: insights from a large multicenter registry. J Am Coll Cardiol. 2013;62(17):1552-1562.

5. Prakash R, Crouch G, Joseph MX, Bennetts J, Selvanayagam JB, Sinhal A. Very late ventricular displacement of transcatheter aortic valve resulting in severe paravalvular regurgitation. JACC Cardiovasc Interv. 2014;7(2):e13-15.

6. Pang PY, Chiam PT, Chua YL, Sin YK. A survivor of late prosthesis migration and rotation following percutaneous transcatheter aortic valve implantation. Eur J Cardiothorac Surg. 2012;41(5):1195-1196.

7. Bhandary SP, Otey AJ, Papadimos TJ, et al. Case report: post transcatheter aortic valve replacement shock: value of multimodal imaging. F1000Research. 2016;5:2032.

8. Dvir D, Leipsic J, Blanke P, Ribeiro HB, Kornowski R, Pichard A, Rodes-Cabau J, et al. Coronary obstruction in transcatheter aortic valve-in-valve implantation: preprocedural evaluation, device selection, protection, and treatment. Circ Cardiovasc Interv. 2015;8(1): e002079.

9. Spiro J, Nadeem A, Doshi SN. Delayed left main stem obstruction following successful TAVI with an Edwards SAPIEN XT valve: successful resuscitation and percutaneous coronary intervention using a non-invasive automated chest compression device (AutoPulse). J Invasive Cardiol. 2012;24(5):224-228.

10. Broyd CJ, Mullen M, Shiu MF. Late Presentation of a semicomplete occluded right coronary artery by a direct flow valve preventing interventional therapy for ST-segment elevation myocardial infarction. JACC Cardiovasc Interv. 2016;9(15):e149-151. 